\title{
Index of a Family of Dirac Operators on Loop Space *
}

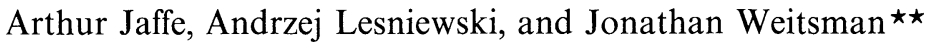 \\ Harvard University, Cambridge, MA 02138, USA
}

Dedicated to Walter Thirring on his $60^{\text {th }}$ birthday

\begin{abstract}
We use methods of constructive field theory to generalize index theory to an infinite-dimensional setting. We study a family of Dirac operators $Q$ on loop space. These operators arise in the context of supersymmetric nonlinear quantum field models with Hamiltonians $H=Q^{2}$. In these models $Q$ is self-adjoint and Fredholm. A natural grading operator $\Gamma$ exists such that $\Gamma Q+Q \Gamma=0$. We study $Q_{+}=P_{-} Q P_{+}$, where $P_{ \pm}=\frac{1}{2}(1 \pm \Gamma)$ are the orthogonal projections onto the eigenspaces of $\Gamma$. We calculate the index $i\left(Q_{+}\right)$for WessZumino models defined by a superpotential $V(\varphi)$. Here $V$ is a polynomial of degree $n \geqq 2$. We establish that $i\left(Q_{+}\right)=n-1=\operatorname{deg} \partial V$. In particular, the field theory models have unbroken supersymmetry, and (for $n \geqq 3$ ) they have degenerate vacua. We believe that this is the first index theorem for a Dirac operator that couples infinitely many degrees of freedom.
\end{abstract}

\section{Introduction}

In this paper we present index theory for a family of Dirac operators on loop space. Since loop space is infinite-dimensional, the mathematical framework requires careful analysis. Each Dirac operator $Q$ which we study will be associated with a stochastic process over loop space. The most interesting such processes are nonGaussian. Our mathematical presentation relies on methods of constructive quantum field theory [1] to define and study the infinite-dimensional processes. We proceed by several steps:

1. We define a family of Dirac operators $Q$ and appeal to a companion paper for mathematical existence theorems [2].

2. For each such $Q$, we introduce a family $Q(\kappa), 0 \leqq \kappa \leqq \infty$, which interpolates between $Q \equiv Q(\infty)$ and $Q(0)=Q_{0}+Q_{i, 0}$. Here $Q_{0}$ is associated with a Gaussian

\footnotetext{
* Research supported in part by the National Science Foundation under Grant DMS/PHY$86-45122$

$\star \star$ Hertz Foundation Predoctoral Fellow
} 
process on loop space and $Q_{i, 0}$ is associated with a non-Gaussian process in a finite-dimensional space.

3. We prove that $Q(\kappa)$ is Fredholm for $0 \leqq \kappa \leqq \infty$, and we show that the deformation from $Q(\infty)$ to $Q(0)$ is a homotopy.

4. By this method we reduce the index theory for $Q$ to index theory for $Q(0)$.

5. We compute the index of $Q(0)$.

The examples we study are motivated by supersymmetric quantum field theory [3-5]. In another paper, we have established the existence of nonlinear supersymmetric interactions in a cylindrical space-time [2]. These models are known in the (formal) physics literature as $N=2$ Wess-Zumino quantum fields. The Hamiltonians for the models have the form $H=Q^{2}$, where $Q$ is a self-adjoint operator.

The operators $H$ and $Q$ are unbounded operators on the Fock space $\mathscr{H}=\mathscr{H}_{b} \otimes \mathscr{H}_{f}$ over the circle (one torus) $T^{1}$. Here $\mathscr{H}_{b}$ and $\mathscr{H}_{f}$ are the bosonic and fermionic Fock spaces. The Atiyah-Singer framework to study the index of the Dirac operator $Q$ requires the introduction of a grading operator $\Gamma$ on the Hilbert space $\mathscr{H}$ [6]. A natural grading of $\mathscr{H}$ is given by $\Gamma=(-1)^{N_{f}}$, where $N_{f}$ is the fermionic particle number operator [3]. The operator $\Gamma$ is self-adjoint and unitary, so $\mathscr{H}$ splits as an orthogonal sum of eigenspaces $\mathscr{H}_{ \pm}$of $\Gamma$,

$$
\mathscr{H}=\mathscr{H}_{+} \oplus \mathscr{H}_{-} .
$$

Let $P_{ \pm}$denote the orthogonal projections onto $\mathscr{H}_{ \pm}$.

A supersymmetric quantum theory is defined by a quadruple $(\mathscr{H}, H, Q, \Gamma)$, where $\mathscr{H}$ is a Hilbert space and $H, Q, \Gamma$ are linear self-adjoint transformations on $\mathscr{H}$. The grading operator $\Gamma$ is unitary and satisfies

$$
Q \Gamma+\Gamma Q=0
$$

from which we conclude $P_{ \pm} Q P_{ \pm}=0$. Thus in terms of the decomposition (I.1), the operators $Q$ and $H=Q^{2}$ have representations

$$
Q=\left(\begin{array}{cc}
0 & Q_{-} \\
Q_{+} & 0
\end{array}\right), \quad H=\left(\begin{array}{cc}
Q_{-} Q_{+} & 0 \\
0 & Q_{+} Q_{-}
\end{array}\right),
$$

where $Q_{+}: \mathscr{H}_{+} \rightarrow \mathscr{H}_{-}$is the adjoint of $Q_{-}$. In our examples, $Q_{+}$will be Fredholm, namely it will be closed and have a closed range, with a finite-dimensional kernel and cokernel. Define

$$
n_{ \pm}=\operatorname{dim} \operatorname{Ker} Q_{ \pm} .
$$

We study the Atiyah-Singer index of $Q$, namely

$$
i\left(Q_{+}\right)=n_{+}-n_{-}
$$

for $Q$ given by the Wess-Zumino potential $V(\varphi)$. Such a $V$ is a holomorphic function of $\varphi \in \mathbb{C}$ taken here to be a polynomial of degree $n \geqq 2$,

$$
V(\varphi)=\frac{1}{2} m \varphi^{2}+\sum_{j=3}^{n} a_{j} \varphi^{j}=\frac{1}{2} m \varphi^{2}+P(\varphi),
$$

with $m>0$ and with $a_{n} \neq 0$ if $n \geqq 3$. It is a remarkable fact that with this structure the field theory defined by $Q$ is finite - all ultraviolet infinites cancel and no renormalization is necessary. We establish this by nonperturbative methods in [2]. 
We prove here:

i) The value of the index is determined by the degree of $\partial \mathrm{V}$,

$$
i\left(Q_{+}\right)=n-1 .
$$

ii) The index has an integral representation

$$
i\left(Q_{+}\right)=\int_{\mathscr{D}^{\prime}\left(T^{2}\right)} \operatorname{det}_{3}(1-K) \exp (-\mathscr{A}) d \mu_{C},
$$

where $d \mu_{C}$ is a Gaussian measure on the space of distributions $\mathscr{D}^{\prime}\left(T^{2}\right)$ on the twotorus, where the determinant is a regularized Fredholm determinant, and where $\mathscr{A}$ is a function on $\mathscr{D}^{\prime}\left(T^{2}\right)$ which depends on $V$.

In particular, (I.7) proves that the ground state energy $E$ of $H$ is identically zero. Furthermore, the ground state is degenerate if $n \geqq 3$. Since $E=0$, supersymmetry is unbroken for all these finite volume models. In spite of the fact that for $n \geqq 3$ the field theory has multiple ground states; the model has a single "phase." In other words, the model is uniquely specified by the parameters $\left\{m, a_{j}\right\}$ of $V$.

We conjecture that the same phenomena illustrated here persist in the infinite volume limit for a subspace of the parameter space $\left\{m, a_{j}\right\}$; we are investigating that question. This appears incompatible with the standard Wightman axioms for quantum fields [7]. In that framework one assumes a unique vacuum, or else the existence of a superselection rule which yields a direct integral decomposition into theories with a unique vacuum. In our case, the Wightman framework needs to be generalized to include the possibilities of the issues raised here.

\section{Dirac Operators on Loop Space}

The Hilbert space $\mathscr{H}$ of our model is a tensor product of the bosonic Hilbert space $\mathscr{H}_{b}$ and the fermionic Hilbert space $\mathscr{H}_{f}$, namely $\mathscr{H}=\mathscr{H}_{b} \otimes \mathscr{H}_{f}$. In both cases we assume that the one particle space is built over the circle (one torus) $T^{1}$ of length $\ell$.

\section{II.1. The Bosonic Fock Space}

The one particle space of the complex scalar field is

$$
W=L_{2}\left(T^{1}\right) \oplus L_{2}\left(T^{1}\right) \equiv W_{+} \oplus W_{-} .
$$

The Fock space $\mathscr{H}_{b}$ is a symmetric tensor algebra over $W$ with the natural inner product yielding on the $n$-fold tensor product $\|f \otimes \ldots \otimes f\|=\|f\|^{n}, f \in W$. In the Fourier space (momentum representation) we define annihilation operators $a_{ \pm}(p)$ on $W_{ \pm}$so that $a_{ \pm} \Omega_{0}^{b}=0, \Omega_{0}^{b}=(1,0, \ldots, 0, \ldots)$, and

$$
\begin{aligned}
& {\left[a_{ \pm}(p), a_{ \pm}(q)\right]=\left[a_{ \pm}(p), a_{\mp}(q)\right]=\left[a_{ \pm}(p), a_{+}^{*}(q)\right]=0,} \\
& {\left[a_{ \pm}(p), a_{ \pm}^{*}(q)\right]=\delta_{p q},}
\end{aligned}
$$
where $p \in \hat{T}^{1} \equiv \frac{2 \pi}{\ell} \mathbb{Z}$ and $\delta_{p q}$ is the Kronecker delta. The time zero field is defined
by

$$
\varphi(x)=(2 \ell)^{-1 / 2} \sum_{p \in \hat{T}^{1}} \omega(p)^{-1 / 2}\left(a_{+}^{*}(p)+a_{-}(-p)\right) e^{-i p x},
$$


where $\omega(p)=\left(p^{2}+m^{2}\right)^{1 / 2}$, and $m>0$. The canonical momentum is

$$
\pi(x)=i(2 \ell)^{-1 / 2} \sum_{p \in \hat{T}^{1}} \omega(p)^{1 / 2}\left(a_{-}^{*}(p)-a_{+}(-p)\right) e^{-i p x} .
$$

The scalar field satisfies the commutation relations

$$
\begin{aligned}
& {[\varphi(x), \varphi(y)]=[\pi(x), \pi(y)]=\left[\pi^{*}(x), \varphi(y)\right]=0,} \\
& {[\pi(x), \varphi(y)]=-i \delta(x-y),}
\end{aligned}
$$

where $\delta(x-y)$ is the Dirac measure.

\section{II.2. The Schrödinger Representation (Loop Space)}

Another, unitarily equivalent, representation of $\mathscr{H}_{b}$ is given by the Schrödinger (or loop space) representation. Let $\mathscr{D}\left(T^{1}\right)$ denote the space of smooth maps (loops) from $T^{1}$ to $\mathbb{C}$, with the topology defined by uniform convergence of each derivative. Let $\mathscr{D}^{\prime}\left(T^{1}\right)$ denote the topological dual, i.e., the space of complex distributions on $T^{1}$. Let $d \mu_{G_{\ell}}$ denote the Gaussian measure on $\mathscr{D}^{\prime}\left(T^{1}\right)$ with mean zero and covariance $G_{\ell}=\left(-d^{2} / d x^{2}+m^{2}\right)^{-1 / 2}$. It is then well known that $\mathscr{H}_{b} \cong L^{2}\left(\mathscr{D}^{\prime}\left(T^{1}\right), d \mu_{G_{\ell}}\right)$, see e.g. [1]. Under this isomorphism $\varphi(x)$ becomes a multiplication operator, and $\pi(x)$ becomes $-i \delta / \delta \varphi(x)$, where $\delta / \delta \varphi(x)$ is the Frechet derivative.

\section{II.3. The Fermionic Fock Space}

The fermionic Fock space $\mathscr{H}_{f}$ is the anti-symmetric tensor algebra over $L^{2}\left(T^{1}\right) \oplus L^{2}\left(T^{1}\right)$. The annihilation operators are $b_{ \pm}(p), p \in \hat{T}^{1}$, and they satisfy

$$
\begin{aligned}
& \left\{b_{ \pm}(p), b_{ \pm}(q)\right\}=\left\{b_{ \pm}(p), b_{\mp}(q)\right\}=\left\{b_{ \pm}(p), b_{\mp}^{*}(q)\right\}=0, \\
& \left\{b_{ \pm}(p), b_{ \pm}^{*}(q)\right\}=\delta_{p q},
\end{aligned}
$$

where $\{\cdot, \cdot\}$ is the anti-commutator. The time zero Fermi fields are defined by

$$
\begin{aligned}
& \psi_{1}(x)=(2 \ell)^{-1 / 2} \sum_{p \in \hat{T}^{1}} \omega(p)^{-1 / 2}\left(v(-p) b_{-}^{*}(p)+v(p) b_{+}(-p)\right) e^{-i p x}, \\
& \psi_{2}(x)=(2 \ell)^{-1 / 2} \sum_{p \in \hat{T}^{1}} \omega(p)^{-1 / 2}\left(v(p) b_{-}^{*}(p)-v(-p) b_{+}(-p)\right) e^{-i p x},
\end{aligned}
$$

where $v(p)=(\omega(p)+p)^{1 / 2}$. Let $\bar{\psi}_{1}(x) \equiv \psi_{2}^{*}(x), \quad \bar{\psi}_{2}(x) \equiv \psi_{1}^{*}(x)$, corresponding to $\bar{\psi}=\psi^{*}\left(\begin{array}{ll}0 & 1 \\ 1 & 0\end{array}\right)$. Then

$$
\begin{aligned}
& \left\{\psi_{\mu}(x), \psi_{\nu}(y)\right\}=0, \quad \mu, v=1,2, \\
& \left\{\bar{\psi}_{\mu}(x), \psi_{\mu}(y)\right\}=0, \quad \mu=1,2, \\
& \left\{\bar{\psi}_{1}(x), \psi_{2}(y)\right\}=\left\{\bar{\psi}_{2}(x), \psi_{1}(y)\right\}=\delta(x-y) .
\end{aligned}
$$

Relations (II.7) mean that $\psi_{\mu}(f) \equiv \int_{T^{1}} \psi_{\mu}(x) f(x) d x$ and $\bar{\psi}_{\mu}(f) \equiv \int_{T^{1}} \bar{\psi}_{\mu}(x) f(x) d x$, for $f \in L^{2}\left(T^{1}\right)$, generate an infinite dimensional Clifford algebra. It also follows from (II.7) that $\psi_{\mu}(f)$ is a bounded operator and $\left\|\psi_{\mu}(f)\right\|=\|f\|_{L^{2}}$. 


\section{II.4. The Operators $N_{\tau}$}

For $0 \leqq \tau \leqq 1$ we define the operators

$$
\begin{aligned}
& N_{\tau, b}=\sum_{j= \pm} \sum_{p \in T^{1}} \omega(p)^{\tau} a_{j}^{*}(p) a_{j}(p), \\
& N_{\tau, f}=\sum_{j= \pm} \sum_{p \in T^{1}} \omega(p)^{\tau} b_{j}^{*}(p) b_{j}(p),
\end{aligned}
$$

on dense subspaces of $\mathscr{H}_{b}$ and $\mathscr{H}_{f}$, respectively. Let

$$
N_{\tau}=N_{\tau, b} \otimes I_{\mathscr{H}_{f}}+I_{\mathscr{H}_{b}} \otimes N_{\tau, f}
$$

be defined on $\mathscr{H}$. Clearly the number operator is $N=N_{0}$ and the free field Hamiltonian is $H_{0}=N_{1}$. For $0<\tau<1$ these $N_{\tau}$ operators interpolate between $N$ and $H_{0}$. It clearly causes no confusion to suppress the tensor products with $I$.

Let us also introduce the involution

$$
\Gamma=\exp \left(i \pi N_{f}\right)
$$

on $\mathscr{H}$, where $N_{f}=N_{0, f}$ is the fermionic number operator. $\Gamma$ induces a grading on $\mathscr{H}$, as described in the Introduction.

Proposition II.1. For $\tau, \beta>0$ the operators $\exp \left(-\beta N_{\tau, b}\right)$ and $\exp \left(-\beta N_{\tau, f}\right)$ are trace class and

$$
\begin{aligned}
\operatorname{Tr}_{\mathscr{H}_{b}}\left(\exp \left(-\beta N_{\tau, b}\right)\right) & =\prod_{p \in T^{1}}\left(1-\exp \left(-\beta \omega(p)^{\tau}\right)\right)^{-2}, \\
\operatorname{Tr}_{\mathscr{H}_{f}}\left(\exp \left(-\beta N_{\tau, f}\right)\right) & =\prod_{p \in T^{1}}\left(1+\exp \left(-\beta \omega(p)^{\tau}\right)\right)^{2}, \\
\operatorname{Tr}_{\mathscr{H}_{f}}\left(\Gamma \exp \left(-\beta N_{\tau, f}\right)\right) & =\prod_{p \in T^{1}}\left(1-\exp \left(-\beta \omega(p)^{\tau}\right)\right)^{2} .
\end{aligned}
$$

Remark. As a corollary to (II.10) and (II.12) we obtain the following identity

$$
\operatorname{Tr}_{\mathscr{H}}\left(\Gamma \exp \left(-\beta N_{\tau}\right)\right)=1
$$

valid for $\tau, \beta>0$.

Proof. Let $\mathscr{H}_{b}(p, j), p \in \hat{T}^{1}, j= \pm$, be the subspace of $\mathscr{H}_{b}$ spanned by polynomials in the creation operator $a_{j}^{*}(p)$ applied to the ground state $\Omega_{0}^{b}$. Then

$$
\mathscr{H}_{b} \cong \bigotimes_{p, j} \mathscr{H}_{b}(p, j)
$$

and

$$
\operatorname{Tr}_{\mathscr{H}_{b}}\left(\exp \left(-\beta N_{\tau, b}\right)\right)=\prod_{p, j} \operatorname{Tr}_{\mathscr{H}_{b}(p, j)}\left(\exp \left(-\omega(p)^{\tau} a_{j}^{*}(p) a_{j}(p)\right) .\right.
$$

The spectrum of $a_{j}^{*}(p) a_{j}(p)$ consists of the eigenvalues $\mathbb{Z}_{+}$, each with multiplicity one. Thus

$$
\operatorname{Tr}_{\mathscr{H}_{b}(p, j)}\left(\exp \left(-\omega(p)^{\tau} a_{j}^{*}(p) a_{j}(p)\right)=\left(1-\exp \left(-\beta \omega(p)^{\tau}\right)\right)^{-1},\right.
$$

which proves (II.10). 
We have

$$
\mathscr{H}_{f} \cong \bigwedge_{p, j} \mathscr{H}_{f}(p, j),
$$

and observe that the spectrum of $b_{j}^{*}(p) b_{j}(p)$ is $\{0,1\}$. The proof of (II.11) and (II.12) follows.

\section{II.5. The Cutoff Interaction}

Let $V$ be given by (I.6). The supercharge $Q$ is defined as a bilinear form on $\mathscr{H}$.

$$
Q=\frac{1}{\sqrt{2}} \int_{T^{1}} d x \psi_{1}\left(\pi-\partial_{1} \varphi^{*}-i \partial V(\varphi)\right)+\psi_{2}\left(\pi^{*}-\partial_{1} \varphi-i \partial V(\varphi)^{*}\right)+\text { h.c. },(I
$$

where h.c. denotes hermitian conjugate. The domain $\mathscr{D}_{0}$ of $Q$ we choose consists of Fock states with finite number of particles and $\mathscr{D}\left(T^{1}\right)$-valued wave functions. Notice that $Q$ has the structure of a Dirac operator on an infinite dimensional manifold [loop space $\left.\mathscr{D}\left(T^{1}\right)\right]$ with circle action. The terms $\partial_{1} \varphi$ and $\partial_{1} \varphi^{*}$ are generators of the circle action $\varphi(x) \rightarrow \varphi(x+y), y \in T^{1}$. Also $\left(\partial V(\varphi), \partial V(\varphi)^{*}\right)$ is the connection of a flat bundle over $\mathscr{D}\left(T^{1}\right)$. Defining (II.15) as an operator on $\mathscr{H}$ requires careful definition of its domain. We first smooth the form $Q$, and then we exhibit cancellation of local (ultraviolet) singularities. Finally we justify removing the smoothing.

We use the following smooth approximation to the periodic Dirac measure

$$
\chi_{\kappa}(x)=\kappa \sum_{n \in \mathbb{Z}} \chi(\kappa(x-n \ell))
$$

where $\kappa>0$ and where $\chi$ satisfies six conditions: $0 \leqq \chi \in \mathscr{S}(\mathbb{R}), \chi(x)=\chi(-x)$, $\int \chi(x) d s=1, \hat{\chi}(p) \geqq 0$, supp $\hat{\chi} \subset[-1,1]$, and $\hat{\chi}(p)>0$ for $|p|<1 / 2$. We define regularized (cutoff) fields by convoluting with $\chi_{\kappa}$ on $T^{1}$,

$$
\varphi_{\kappa}(x)=\chi_{\kappa} * \varphi(x), \quad \psi_{\mu, \kappa}(x)=\chi_{\kappa} * \psi_{\mu}(x) .
$$

The regularized supercharge $Q(\kappa)$ is defined as a bilinear form on $\mathscr{H}$,

$$
Q(\kappa)=Q_{0}+Q_{i, \kappa},
$$

where

$$
Q_{0}=\frac{1}{\sqrt{2}} \int_{T^{1}} d x \psi_{1}\left(\pi-\partial_{1} \varphi^{*}-i m \varphi\right)+\psi_{2}\left(\pi^{*}-\partial_{1} \varphi-i m \varphi^{*}\right)+\text { h.c. }
$$

and

$$
Q_{i, \kappa}=-\frac{i}{\sqrt{2}} \int_{T^{1}} d x \psi_{1} \partial P\left(\varphi_{\kappa}\right)+\psi_{2} \partial P\left(\varphi_{\kappa}\right)^{*}+\text { h.c. },
$$

where $P(\varphi)$ is the sum of the $j \geqq 3$ terms in (I.6).

Proposition II.2. The form $Q(\kappa)$ defines a symmetric operator with domain $\mathscr{D}_{0}$, such that (as a form) its square equals

$$
\begin{aligned}
H(\kappa) \equiv Q(\kappa)^{2}=H_{0} & +\int_{T^{1}} d x\left(m \varphi^{*} \partial P\left(\varphi_{\kappa}\right)-\left(\bar{\psi}_{1} \psi_{1}\right)_{\kappa} \partial^{2} P\left(\varphi_{\kappa}\right)+\text { h.c. }\right) \\
& +\int_{T^{1}} d x\left|\partial P\left(\varphi_{\kappa}\right)\right|^{2} .
\end{aligned}
$$


Here $\left(\bar{\psi}_{\mu} \psi_{\mu}\right)_{\kappa} \equiv \frac{1}{2}\left(\bar{\psi}_{\mu, \kappa} \psi_{\mu}+\bar{\psi}_{\mu} \psi_{\mu, \kappa}\right)$. Thus $H(\kappa)$ extends uniquely to a symmetric operator with domain $\mathscr{D}_{0}$.

Proof. The form $Q_{0}$ uniquely defines an operator $Q_{0}$ on $\mathscr{D}_{0}$. Furthermore, $Q_{0}^{2}=H_{0}$. In fact, we have the explicit representation:

$$
Q_{0}^{2}=\int_{T^{1}} d x H_{0}(x)
$$

where

$$
H_{0}(x)=\lim _{\kappa \rightarrow \infty}\left\{\left|\pi_{\kappa}(x)\right|^{2}+\left|\partial_{1} \varphi_{\kappa}(x)\right|^{2}+m^{2}\left|\varphi_{\kappa}(x)\right|^{2}+\bar{\psi}_{\kappa}(x)\left(i \gamma_{1} \partial_{1}-m\right) \psi_{\kappa}(x)\right\},
$$

and where $\gamma_{1}=\left(\begin{array}{rr}0 & -1 \\ 1 & 0\end{array}\right)$. The Wick ordering constants of the bosonic and fermionic parts cancel identically in the sum, and we can also write

$$
\begin{aligned}
H_{0}(x) & =:|\pi(x)|^{2}+\left|\partial_{1} \varphi(x)\right|^{2}+m^{2}|\varphi(x)|^{2}:+: \bar{\psi}(x)\left(i \gamma_{1} \partial_{1}-m\right) \psi(x): \\
& =H_{0, b}(x)+H_{0, f}(x),
\end{aligned}
$$

where $H_{0, b}(x)$ and $H_{0, f}(x)$ are the densities for $H_{0, b}$ and $H_{0, f}$, respectively. The remaining terms in $Q(\kappa)^{2}$ are well behaved on $\mathscr{D}_{0} \times \mathscr{D}_{0}$. Elementary but lengthy application of the commutation and anti-commutation relations yields (II.20). In fact, each term in (II.20) defines an operator on $\mathscr{D}_{0}$, so $H(\kappa)$ is a symmetric operator on that domain.

\section{II.6. The Zero Momentum Mode}

Set

$$
\varphi_{0}=\ell^{-1 / 2} \hat{\varphi}(0), \quad \psi_{\mu, 0}=\ell^{-1 / 2} \hat{\psi}_{\mu}(0),
$$

where $\hat{\varphi}(p)=\ell^{-1 / 2} \int_{T^{1}} d x \varphi(x) e^{i p x}$. Define

$$
Q(0)=Q_{0}+Q_{i, 0},
$$

where

$$
Q_{i, 0}=\frac{i}{\sqrt{2}} \ell\left(\psi_{1,0} \partial P\left(\varphi_{0}\right)+\psi_{2,0} \partial P\left(\varphi_{0}\right)^{*}\right)+\text { h.c. . }
$$

We also set $H(0)=Q(0)^{2}$. Here $H(0)$ is the Hamiltonian of a theory where the only interacting mode is the zero mode.

\section{II.7. The Cutoff Removed}

In this subsection we formulate two results of a technical character, which we prove in a separate paper [2].

Theorem II.3. (i) The operators $Q(\kappa)$ and $H(\kappa)$ are essentially self-adjoint on the domain $\mathscr{D}_{0}$ for all $0 \leqq \kappa<\infty$.

(ii) The resolvents of their closures converge in the operator norm as $\kappa \rightarrow \infty$ to the resolvents of self-adjoint operators $Q$ and $H$ respectively. 
(iii) Define $Q(\infty) \equiv Q, H(\infty) \equiv H$. The mappings $\kappa \rightarrow \operatorname{Resolvent}(Q(\kappa))$ and $\kappa \rightarrow \operatorname{Resolvent}(H(\kappa))$ are continuous in the operator norm for $0 \leqq \kappa \leqq \infty$.

Remark. The theorem suggests that $\kappa \rightarrow Q(\kappa)$ is a homotopy interpolating between the field theory supercharge $Q$ and the degenerate supercharge $Q(0)$ which involves only one interacting mode. We demonstrate this fact in Theorem II.6.

Theorem II.4. Let $\tau \in(0,1)$ be fixed. There exist constants $\zeta>0, C<\infty$ such that

$$
\zeta N_{\tau} \leqq H(\kappa)+C
$$

uniformly in $0 \leqq \kappa \leqq \infty$.

Since $N_{\tau}$ has a compact resolvent for $\tau>0$, this crucial estimate yields

Corollary II.5. The resolvents of $H(\kappa)$ and $Q(\kappa), 0 \leqq \kappa \leqq \infty$, are compact.

\section{II.8. The Index}

Recall that a densely-defined, closed operator is called Fredholm if its range is closed, and its kernel and its cokernel are finite-dimensional. Let Fred $\left(\mathscr{H}_{+}, \mathscr{H}_{-}\right)$ denote the set of Fredholm operators from $\mathscr{H}_{+}$to $\mathscr{H}_{-}$, and let $Q_{ \pm}(\kappa)=P_{\mp} Q(\kappa) P_{ \pm}$ (cf. Sect. I).

Theorem II.6. For $0 \leqq \kappa \leqq \infty, Q_{+}(\kappa) \in \operatorname{Fred}\left(\mathscr{H}_{+}, \mathscr{H}_{-}\right)$. The index $i\left(Q_{+}(\kappa)\right)$ is constant for $0 \leqq \kappa \leqq \infty$.

Proof. By Corollary II.5, the resolvent of $Q(\kappa)$ is compact. Thus the kernel of $Q(\kappa)$ is finite-dimensional. Using (I.3), $\operatorname{Ker}(Q(\kappa))=\operatorname{Ker}\left(Q_{+}(\kappa)\right) \oplus \operatorname{Ker}\left(Q_{-}(\kappa)\right)$, so $\operatorname{dim} \operatorname{Ker}\left(Q_{ \pm}(\kappa)\right)<\infty$. Furthermore,

$$
(Q(\kappa)+i)^{-1} Q(\kappa)=1-i(Q(\kappa)+i)^{-1} .
$$

Since $(Q(\kappa)+i)^{-1}$ is compact, standard results of functional analysis imply that the range of $Q(\kappa)$ is closed. This implies that the range of $Q_{+}(\kappa)$ is closed, and so $Q_{+}(\kappa) \in \operatorname{Fred}\left(\mathscr{H}_{+}, \mathscr{H}_{-}\right)$. Since $\kappa \rightarrow \operatorname{Resolvent}(Q(\kappa)) \quad$ is norm-continuous, Theorem IV.5.17 of [8] implies that $i\left(Q_{+}(\kappa)\right)$ is locally constant (and thus constant).

Proposition II.7. For $0 \leqq \kappa \leqq \infty, e^{-\beta H(\kappa)}$ is trace class, and

$$
i\left(Q_{+}(\kappa)\right)=\operatorname{Tr}\left(\Gamma e^{-\beta H(\kappa)}\right) .
$$

where $\mathrm{Tr}$ denotes the trace on $\mathscr{H}_{b} \otimes \mathscr{H}_{f}$.

Proof. Formula (II.25) is the well-known heat kernel representation of the index, see e.g. [9]. The only nontrivial statement is that $\exp (-\beta H(\kappa))$ is trace class. This follows from Proposition II.1 and the comparison estimate (II.24).

\section{Computation of the Index}

In the preceding section we showed that the estimates of [2] are sufficient to establish a homotopy between $Q(\infty)$ and $Q(0)$ with $i\left(Q_{+}(\kappa)\right)$ constant. Here we evaluate $i\left(Q_{+}(0)\right)$. 
Theorem III.1 (index theorem). Let $V(\varphi)=\frac{1}{2} m \varphi^{2}+P(\varphi)$ be a polynomial of the form (I.6), and let $Q$ be the corresponding supercharge. Then $i\left(Q_{+}\right)=n-1$.

Proof. By Theorem II.6, we need only consider the case $\kappa=0$. Decompose the Fock space as an orthogonal sum $\mathscr{H}=\mathscr{H}_{0} \oplus \mathscr{H}_{0}^{\perp}$, where $\mathscr{H}_{0}$ is the subspace spanned by the zero-momentum modes. According to this decomposition, the free supercharge $Q_{0}$ can be written

$$
Q_{0}=\left(\begin{array}{cc}
Q_{0}^{0} & 0 \\
0 & Q_{0}^{\perp}
\end{array}\right)
$$

where the zero modes contribute

$$
\begin{aligned}
Q_{0}^{0}= & \frac{1}{\sqrt{2}}\left(\left(\hat{\psi}_{1}(0)+\hat{\bar{\psi}}_{1}(0)\right) \hat{\pi}(0)+\left(\hat{\psi}_{2}(0)+\hat{\bar{\psi}}_{2}(0)\right) \hat{\pi}^{*}(0)\right. \\
& \left.+i m \hat{\varphi}(0)\left(\hat{\bar{\psi}}_{1}(0)-\hat{\psi}_{1}(0)\right)+i m \hat{\varphi}^{*}(0)\left(\hat{\bar{\psi}}_{2}(0)-\hat{\psi}_{2}(0)\right)\right) .
\end{aligned}
$$

Introduce new variables $z=\hat{\varphi}(0)$ and

$$
\begin{array}{ll}
\Psi_{1}=-\frac{1}{2}\left(b_{-}^{*}(0)+b_{+}(0)-b_{-}(0)+b_{+}^{*}(0)\right), & \bar{\Psi}_{2}=\Psi_{1}^{*}, \\
\Psi_{2}=-\frac{1}{2}\left(b_{-}^{*}(0)-b_{+}(0)+b_{-}(0)+b_{+}^{*}(0)\right), & \bar{\Psi}_{1}=\Psi_{2}^{*} .
\end{array}
$$

Then we verify

$$
\begin{aligned}
& \left\{\Psi_{\mu}, \Psi_{v}\right\}=0, \quad \mu, v=1,2, \\
& \left\{\Psi_{\mu}, \bar{\Psi}_{\mu}\right\}=0, \quad \mu=1,2, \\
& \left\{\Psi_{1}, \bar{\Psi}_{2}\right\}=\left\{\Psi_{2}, \bar{\Psi}_{1}\right\}=1 .
\end{aligned}
$$

In terms of these variables

$$
Q_{0}^{0}=i \bar{\Psi}_{1}(\partial / \partial z)+i \Psi_{2}(\partial / \partial \bar{z})+i \Psi_{1} m z-i \bar{\Psi}_{2} m \bar{z} .
$$

The operator $Q_{i, 0}$ can also be expressed as an operator on $\mathscr{H}_{0}$, namely

$$
Q_{i, 0}=i \ell^{1 / 2} \Psi_{1} \partial P\left(\ell^{-1 / 2} z\right)-i \ell^{1 / 2} \bar{\Psi}_{2} \partial P\left(\ell^{-1 / 2} z\right)^{*} .
$$

Therefore

$$
\begin{aligned}
Q_{0}^{0}+Q_{i, 0}= & i \bar{\Psi}_{1}(\partial / \partial z)+i \Psi_{2}(\partial / \partial \bar{z}) \\
& +i \ell^{1 / 2} \Psi_{1} \partial V\left(\ell^{-1 / 2} z\right)-i \ell^{1 / 2} \bar{\Psi}_{2} \partial V\left(\ell^{-1 / 2} z\right)^{*} .
\end{aligned}
$$

Comparing (III.6) with Sect. 4 of [10], we find that $Q_{0}^{0}+Q_{i, 0}$ is exactly the supercharge of our model of holomorphic quantum mechanics. In addition, we verify by explicit calculation that $\Gamma \uparrow_{\mathscr{H}_{0}}$ is identical to the operator $\gamma=\gamma_{0} \gamma_{1} \gamma_{2} \gamma_{3}$ in formula (4.3) of [10]. Thus the index calculation for $\left(Q_{0}^{0}+Q_{i, 0}\right)+$ reduces to the calculation of $[10]$, where we established $n_{+}=n-1, n_{-}=0$. On the full Fock space

$$
Q(0)=\left(\begin{array}{cc}
Q_{0}^{0}+Q_{i, 0} & 0 \\
0 & Q_{0}^{\perp}
\end{array}\right) .
$$

Clearly $Q_{0}^{\perp}$ has a unique ground state which is an element of $\mathscr{H}_{+}$; it has no ground state in $\mathscr{H}_{-}$. This completes the proof of the theorem. 
Remark. Since $n_{ \pm}(\kappa)$ are not homotopy invariants, this argument does not suffice to conclude that $n_{-}(\kappa)=0$ ("vanishing theorem") for $\kappa>0$. We conjecture that $n_{-}(\kappa)=0$ holds for all $\kappa \geqq 0$.

\section{Path Integrals and the Index}

In this section we establish Feynman-Kac representations for the trace states defined by the heat kernels of our Hamiltonian. These states are also called "finite temperature" states since they correspond to Gibbs states in quantum statistical mechanics with inverse temperatures $\beta$. These representations complement the Feynman-Kac representation of the vacuum functional established in [2], and the latter are recovered in the limit $\beta \rightarrow \infty$. Special cases of these representations were derived in [11] and also in the formal physics literature.

The special feature of the finite temperature states is the fact that the underlying Gaussian functional integrals are replaced by Gaussian integrals with boundary conditions in time. In this way the cylinder is replaced by a torus with period $\beta$ in the time direction. The bosonic integral has periodic boundary conditions with period $\beta$. For the fermionic integral, however, there are two different cases. The pure trace state is represented by antiperiodic boundary conditions in the time direction. The graded (super) trace, on the other hand, gives rise to periodic boundary conditions in the time direction. Our study requires both types of states: the pure trace to establish regularity estimates and the super trace to represent the index.

\section{IV.1. Path Integral Representation of the Index}

We define the "finite temperature" free states:

$$
\begin{aligned}
\langle\cdot\rangle_{\beta, b}=\left\{\operatorname{Tr}_{\mathscr{H}_{b}}\left(\exp \left(-\beta H_{0, b}\right)\right)\right\}^{-1} \operatorname{Tr}_{\mathscr{H}_{b}}\left(\cdot \exp \left(-\beta H_{0, b}\right)\right), \\
\langle\cdot\rangle_{\beta, f}^{(k)}=\left\{\operatorname{Tr}_{\mathscr{H}_{f}}\left(\Gamma^{k} \exp \left(-\beta H_{0, f}\right)\right)\right\}^{-1} \operatorname{Tr}_{\mathscr{H}_{f}}\left(\cdot \Gamma^{k} \exp \left(-\beta H_{0, f}\right)\right), \quad k=0,1,
\end{aligned}
$$

with $\Gamma$ given by (II.9). Here $\Gamma^{k}$ denotes $\Gamma$ to the power zero or one. We set $C_{\ell, \beta}=\left(-\Delta+m^{2}\right)^{-1}$, where $\Delta$ is the Laplace operator on the torus $T^{2}=\mathbb{R}^{2} / \ell \mathbb{Z} \times \beta \mathbb{Z}$. In other words, $C_{\ell, \beta}$ has periodic boundary conditions with period $\ell$ in the space direction and period $\beta$ in the time direction. Let $\not D=i \gamma_{0}^{E} \partial_{0}+i \gamma_{1}^{E} \partial_{1}$ be the Euclidean Dirac operator on $T^{2}$, where the Euclidean Dirac matrices $\gamma_{\mu}^{E}$ are

$$
\gamma_{0}^{E}=\left(\begin{array}{cc}
0 & -i \\
-i & 0
\end{array}\right), \quad \gamma_{1}^{E}=\left(\begin{array}{cc}
0 & -1 \\
1 & 0
\end{array}\right) .
$$

We set $S_{\ell, \beta}=(\not D+m)^{-1}$ as the periodic Green's function of the Dirac operator.

By $\widetilde{D}$ we denote the Dirac operator on the torus twisted in the time direction by $\pi$. This twisting is interpreted as introducing boundary conditions for the Dirac operator which are anti-periodic in the time direction. Functions in the domain of $\widetilde{D}$ satisfy

$$
f\left(x_{0}+\beta, x_{1}\right)=-f\left(x_{0}, x_{1}\right)
$$


Thus we actually replace the period $\beta$ of the torus in the time direction by a period $2 \beta$, and we impose (IV.3). We define $\tilde{S}_{\ell, \beta}=(\tilde{D}+m)^{-1}$ as the antiperiodic Green's function in the time direction and periodic in the space direction. Finally, let $\mathscr{H}_{\alpha}\left(T^{1}\right)$ denote the Sobolev space of order $\alpha$ over $T^{1}$, namely the completion of $\mathscr{D}\left(T^{1}\right)$ in the norm $\|f\|_{\alpha}=\left\|\left(-\Delta+m^{2}\right)^{\alpha / 2} f\right\|_{L_{2}}$.

Lemma IV.1. Let $s \leqq t$. Then

(i) The finite temperature free bosonic state is given by a Gaussian measure with the periodic covariance $C_{\ell, \beta}$.

$$
\begin{aligned}
& \left\langle e^{-s H_{0, b}} \varphi(f) e^{-(t-s) H_{0, b}} \varphi^{*}(g) e^{t H_{0, b}}\right\rangle_{\beta, b} \\
& \quad=C_{\ell, \beta}\left(f \otimes \delta_{s}, g \otimes \delta_{t}\right), \quad \text { for } \quad f, g \in \mathscr{H}_{-1}\left(T^{1}\right) .
\end{aligned}
$$

(ii) The finite temperature graded (super) free fermionic state is given by a Gaussian fermionic state with covariance $S_{\ell, \beta}$.

$$
\begin{aligned}
& \left\langle e^{-s H_{0, f}} \psi_{\mu}(f) e^{-(t-s) H_{0, f}} \bar{\psi}_{v}(g) e^{t H_{0, f}}\right\rangle_{\beta, f}^{(1)} \\
& \quad=\left(S_{\ell, \beta}\right)_{\mu v}\left(f \otimes \delta_{s}, g \otimes \delta_{t}\right), \quad \text { for } \quad f, g \in \mathscr{H}_{-1 / 2}\left(T^{1}\right) .
\end{aligned}
$$

(iii) The finite temperature (ungraded) free fermionic state is given by a Gaussian fermionic state with covariance $\widetilde{S}_{\ell, \beta}$.

$$
\begin{aligned}
& \left\langle e^{-s H_{0, f}} \psi_{\mu}(f) e^{-(t-s) H_{0, f}} \bar{\psi}_{v}(g) e^{t H_{0, f}}\right\rangle_{\beta, f}^{(0)} \\
& \quad=\left(\tilde{S}_{\ell, \beta}\right)_{\mu, v}\left(f \otimes \delta_{s}, g \otimes \delta_{t}\right), \quad \text { for } \quad f, g \in \mathscr{H}_{-1 / 2}\left(T^{1}\right) .
\end{aligned}
$$

Proof. It is sufficient to check that the covariance operators (IV.4-6) are correct, as the free, finite-temperature states are well known to be Gaussian with mean zero. (i). We represent $\mathscr{H}_{b}$ as (II.13). Straightforward calculations show that

$$
\begin{gathered}
\operatorname{Tr}_{\mathscr{C}_{b}(p, j)}\left(a_{ \pm}^{*}(p) e^{-\sigma H_{0, b}(p, j)} a_{ \pm}(p) e^{-(\beta-\sigma) H_{0, b}(p, j)}\right) \\
=(1-\exp (-\beta \omega(p)))^{-2} \exp (-(\beta-\sigma) \omega(p)),
\end{gathered}
$$

and

$$
\begin{aligned}
& \operatorname{Tr}_{\mathscr{H}_{b}(p, j)}\left(a_{ \pm}(p) e^{-\sigma H_{0, b}(p, j)} a_{ \pm}^{*}(p) e^{-(\beta-\sigma) H_{0, b}(p, j)}\right) \\
& \quad=(1-\exp (-\beta \omega(p)))^{-2} \exp (-\sigma \omega(p)),
\end{aligned}
$$

where $\sigma=t-s$ and $H_{0, b}(p, j)=\omega(p) a_{j}^{*}(p) a_{j}(p)$. The left-hand side of (IV.4) can be thus written as

$$
\begin{aligned}
\sum_{p \in \hat{T}^{1}} & \hat{f}(p) \hat{g}(p)(2 \omega(p))^{-1}(1-\exp (-\beta \omega(p)))^{-1}(\exp (-\sigma \omega(p))+\exp (-(\beta-\sigma) \omega(p))) \\
\quad= & \sum_{n \in \mathbb{Z}} \sum_{p \in T^{1}} \hat{f}(p) \hat{g}(p)(2 \omega(p))^{-1} \exp (-|\sigma+n \beta| \omega(p)) \\
= & \sum_{n \in \mathbb{Z}} \sum_{p \in \hat{T}^{1}} \hat{f}(p) \hat{g}(p) \frac{1}{2 \pi} \int_{-\infty}^{\infty} \frac{1}{E^{2}+p^{2}+m^{2}} e^{i(\sigma+n \beta) E} d E \\
= & C_{\ell, \beta}\left(f \otimes \delta_{s}, g \otimes \delta_{t}\right) .
\end{aligned}
$$

(ii) and (iii). We use (II.14). A calculation shows that

$$
\begin{aligned}
& \operatorname{Tr}_{\mathscr{H}_{f}(p, j)}\left(\Gamma_{p, j}^{k} b_{ \pm}^{*}(p) e^{-\sigma H_{0, f}(p, j)} b_{ \pm}(p) e^{-(\beta-\sigma) H_{0, f}(p, j)}\right) \\
& \quad=(-1)^{k} \exp (-(\beta-\sigma) \omega(\varrho)),
\end{aligned}
$$


and

$$
\begin{aligned}
& \operatorname{Tr}_{\mathscr{H}_{f}(p, j)}\left(\Gamma_{p, j}^{k} b_{ \pm}(p) e^{-\sigma H_{0, f}(p, j)} b_{ \pm}^{*}(p) e^{-(\beta-\sigma) H_{0, f}(p, j)}\right) \\
& \quad=\exp (-\sigma \omega(p)),
\end{aligned}
$$

where $\Gamma_{p, j}=\exp \left(i \pi b_{j}^{*}(p) b_{j}(p)\right)$, and the proof of (IV.5) and (IV.6) now follows as in the bosonic case.

We introduce the following notation. For $\Phi \in \mathscr{D}^{\prime}\left(T^{2}\right)$, set

$$
A_{\ell, \beta}^{(\kappa)}(\Phi)=\int_{T^{2}} d x\left(\left|\partial P\left(\Phi_{\kappa}\right)\right|^{2}+m \Phi \partial P\left(\Phi_{\kappa}\right)^{*}+m \Phi^{*} \partial P\left(\Phi_{\kappa}\right)\right)
$$

where

$$
\Phi_{\kappa}\left(x_{0}, x_{1}\right)=\int_{T^{1}} d x_{1}^{\prime} \Phi\left(x_{0}, x_{1}^{\prime}\right) \chi_{\kappa}\left(x_{1}-x_{1}^{\prime}\right),
$$

and where $\chi_{\kappa}$ is given by (II.16). Let $K_{\ell, \beta}^{(\kappa)}(\Phi)$ be the operator whose integral kernel is

$$
\begin{aligned}
K_{\ell, \beta}^{(\kappa)}(\Phi)(x, y)= & \frac{1}{2} \int_{T^{1}} d z_{1}\left(\left[S_{\ell, \beta}(x-z) \partial^{2} P\left(\Phi_{\kappa}(z)\right) \chi_{\kappa}\left(z_{1}-y_{1}\right)\right.\right. \\
& \left.+S_{\ell, \beta}(x-z) \chi_{\kappa}\left(z_{1}-y_{1}\right) \partial^{2} P\left(\Phi_{\kappa}(y)\right)\right] \Lambda_{+} \\
& +\left[S_{\ell, \beta}(x-z) \partial^{2} P\left(\Phi_{\kappa}(z)\right)^{*} \chi_{\kappa}\left(z_{1}-y_{1}\right)\right. \\
& \left.\left.+S_{\ell, \beta}(x-z) \chi_{\kappa}\left(z_{1}-y_{1}\right) \partial^{2} P\left(\Phi_{\kappa}(y)\right)^{*}\right] \Lambda_{-}\right)
\end{aligned}
$$

where $\Lambda_{+}=\left(\begin{array}{ll}1 & 0 \\ 0 & 0\end{array}\right)$ and $\Lambda_{-}=\left(\begin{array}{ll}0 & 0 \\ 0 & 1\end{array}\right)$ are the chiral projection, and where the time component of $z$ is $z_{0}=y_{0}$, i.e., $z=\left(y_{0}, z_{1}\right)$. Propositions II.1, II.7, Lemma IV.1, and standard approximation arguments (see, e.g., [11-13,2]) yield a Feynman-Kac formula based on the Gaussian measure $d \mu_{C_{\ell, \beta}}$ with mean zero and covariance $C_{\ell, \beta}$.

Theorem IV.2. The index $i\left(Q_{+}(\kappa)\right), 0 \leqq \kappa<\infty$, has the following path integral representation

$$
i\left(Q_{+}(\kappa)\right)=\int_{\mathscr{D}^{\prime}\left(T^{2}\right)} d \mu_{C_{\ell, \beta}}(\Phi) \operatorname{det}\left(1-K_{\ell, \beta}^{(\kappa)}(\Phi)\right) \exp \left(-A_{\ell, \beta}^{(\kappa)}(\Phi)\right),
$$

where det means Fredholm determinant.

Remark 1. A formal version of (IV.9) was given in [4].

Remark 2. By means of Theorem II.6, $i\left(Q_{+}(\kappa)\right)$ is independent of $\kappa$ and it is equal to $i\left(Q_{+}\right)$. The right-hand side of (IV.9) also has a limit as $\kappa \rightarrow \infty$, but in this limit divergences cancel between the Fredholm determinant and $A_{\ell, \beta}^{(\kappa)}$. Using the results of [2], we obtain the following representation of $i\left(Q_{+}\right)$:

$$
i\left(Q_{+}\right)=\int_{\mathscr{D}^{\prime}\left(T^{2}\right)} d \mu_{C_{\ell, \beta}}(\Phi) \operatorname{det}_{3}\left(1-K_{\ell, \beta}(\Phi)\right) \exp \left(-\mathscr{A}_{\ell, \beta}(\Phi)\right),
$$

where $\operatorname{det}_{n}$ is the regularized Fredholm determinant, and where $K_{\ell, \beta}(\Phi)$ $=\lim _{\kappa \rightarrow \infty} K_{\ell, \beta}^{(\kappa)}(\Phi), \mathscr{A}_{\ell, \beta}(\Phi)=\lim _{\kappa \rightarrow \infty}\left[A_{\ell, \beta}^{(\kappa)}(\Phi)+\operatorname{Tr}\left(K_{\ell, \beta}^{(\kappa)}(\Phi)\right)+\frac{1}{2} \operatorname{Tr}\left(K_{\ell, \beta}^{(\kappa)}(\Phi)^{2}\right)\right]$. The representation (IV.10) has the property that $\operatorname{both}_{\operatorname{det}_{3}}\left(I-K_{\ell, \beta}(\Phi)\right)$ and $\exp \left(-\mathscr{A}_{\ell, \beta}(\Phi)\right)$ exist. 


\section{IV.2. Path Integral Computation of the Index}

Below we present an alternative proof of Theorem III.1 which is based on the path integral representation of the index. The advantage of this method is, however, that it is quite general and may be applied to situations in which a direct computation of the index is impossible.

From Theorems II.6 and IV.2, we infer that we can set $\kappa=0$ in (IV.9) to obtain

$$
i\left(Q_{+}\right)=\int_{\mathscr{D}^{\prime}\left(T^{2}\right)} d \mu_{C_{\ell, \beta}}(\Phi) \operatorname{det}\left(1-K_{\ell, \beta}^{(0)}(\Phi)\right) \exp \left(-A_{\ell, \beta}^{(0)}(\Phi)\right) .
$$

We have under Fourier transform the isomorphism

$$
d \mu_{C_{\ell, \beta}} \cong \bigotimes_{p_{1} \in \hat{T}_{1}} d \mu_{\left(-d^{2} / d x_{0}^{2}+p_{1}^{2}+m^{2}\right)^{-1}}
$$

Using this tensor product representation and the fact that $\hat{\chi}_{\kappa=0}(p)=\ell^{-1 / 2} \delta_{p 0}$, we conclude that the integral (IV.11) can be expressed as an integral with respect to



$$
\Phi_{0}\left(x_{0}\right)=\frac{1}{(\beta \ell)^{1 / 2}} \sum_{p_{0} \in \hat{T}^{1}} \hat{\Phi}\left(p_{0}, 0\right) e^{-i p_{0} x_{0}},
$$

where $T^{1}$ is here a circle of length $\beta$, and let

$$
A_{\beta}\left(\Phi_{0}\right)=\ell \int_{T^{1}} d x\left\{\left|\partial P\left(\Phi_{0}\right)\right|^{2}+m \Phi_{0} \partial P\left(\Phi_{0}\right)^{*}+m \Phi_{0}^{*} \partial P\left(\Phi_{0}\right)\right\} .
$$

Also let

$$
K_{\beta}\left(\Phi_{0}\right)(x, y)=\ell^{-1} S_{\beta}(x-z) \partial^{2} P\left(\Phi_{0}(z)\right) \Lambda_{+}+\ell^{-1} S_{\beta}(x-z) \partial^{2} P\left(\Phi_{0}(z)\right)^{*} \Lambda_{-},
$$

where $S_{\beta}=\left(i \gamma_{0}^{E} \partial_{0}+m\right)^{-1}$. Then (IV.11) takes the form

$$
\int_{\mathscr{D}^{\prime}\left(T^{1}\right)} d \mu_{\left(-d^{2} / d x_{0}^{2}+m^{2}\right)^{-1}}\left(\Phi_{0}\right) \operatorname{det}\left(1-K_{\beta}\left(\Phi_{0}\right)\right) \exp \left(-A_{\beta}\left(\Phi_{0}\right)\right) .
$$

We infer from Proposition II.7 that the right-hand side of (IV.9) [and, therefore, also (IV.12)] is independent of $\beta>0$, and thus (IV.12) is equal to its $\beta \downarrow 0$ limit. This limit was evaluated in Theorem 2.2 of [14], and it was found to be equal to $\operatorname{deg} \partial V=n-1$. This proves Theorem III.1.

Remark. It is possible to compute (IV.9) without resorting to the results of [14]. Using Euclidean invariance, we show that the representation (IV.10) is independent of $\ell$. We rotate the coordinates by the angle $\pi / 2$, and use the fact that the righthand side of (IV.10) is invariant under such a rotation. In this manner we exchange the roles of $\ell$ and $\beta$; from the independence of $\beta$ we infer that $i\left(Q_{+}\right)$is independent of $\ell$. We set now $\ell=\beta=\varepsilon$, and let $\varepsilon$ tend to zero in (IV.9). In this limit only the zero momentum modes contribute to the function space integral. We have carried through the argument to justify this dominance, as formally proposed in [5], though we omit the details. The zero momentum modes contribute

$$
i\left(Q_{+}\right)=\frac{2 i}{\pi} \int\left|\partial^{2} V\right|^{2} \exp \left(-|\partial V|^{2}\right) d z d \bar{z}=n-1,
$$

namely the degree of $\partial V$, as claimed. 


\section{References}

1. Glimm, J., Jaffe, A.: Quantum physics. Berlin, Heidelberg, New York: Springer 1987

2. Jaffe, A., Lesniewski, A., Weitsman, J.: The two dimensional, $N=2$, Wess-Zumino model on a cylinder (to appear)

3. Witten, E.: Constraints on supersymmetry breaking. Nucl. Phys. B 202, 253-316 (1982); Witten, E.: Dynamical breaking of supersymmetry. Nucl. Phys. B 188, 513-554 (1981)

4. Cecotti, S., Girardello, L.: Functional measure, topology, and dynamical supersymmetry breaking. Phys. Lett. 110 B, 39-43 (1982);

Cecotti, S., Girardello, L.: Stochastic and parastochastic aspects of supersymmetric functional measures: a new nonperturbative approach to supersymmetry. Ann. Phys. 145, 81-99 (1983)

5. Girardello, L., Imbimbo, C., Mukhi, S.: On constant configurations and the evaluation of the Witten index. Phys. Lett. 132 B, 69-74 (1983)

6. Atiyah, M.F., Singer, I.M.: The index of elliptic operators. III. Ann. Math. 87, 546-604 (1968)

7. Streater, R., Wightman, A.: PCT, spin and statistics, and all that. New York: Benjamin 1964

8. Kato, T.: Perturbation theory for linear operators Berlin, Heidelberg, New York: Springer 1984

9. Gilkey, P.: Invariance theory, the heat equation, and the Atiyah-Singer index theorem. Wilmington Del.: Publish or Perish 1984

10. Jaffe, A., Lesniewski, A., Lewenstein, M.: Ground state structure in supersymmetric quantum mechanics. Ann. Phys. (to appear)

11. Hoegh-Krøhn, R.: Relativistic quantum statistical mechanics in two-dimensional space-time. Commun. Math. Phys. 38, 195-224 (1974)

12. Seiler, E., Simon, B.: Nelson's symmetry and all that in the Yukawa 2 and $\left(\phi^{4}\right)_{3}$ field theories. Ann. Phys. 97, 470-518 (1976)

13. Simon, B.: The $P(\phi)_{2}$ Euclidean (quantum) field theory. Princeton, N.J.: Princeton University Press 1974

14. Getzler, E.: The degree of the Nicolai map in supersymmetric quantum mechanics. J. Funct. Anal. 73 (1987)

Communicated by E. Lieb

Received May 6, 1987 